\title{
Focus assessed transthoracic echocardiography (FATE) to diagnose pleural effusions causing haemodynamic compromise
}

\author{
Nils Petter Oveland ${ }^{1,2^{*}}$, Nigussie Bogale ${ }^{3}$, Benedict Waldron ${ }^{4}$, Kasper Bech $^{4}$, Erik Sloth $^{4,5}$ \\ ${ }^{1}$ Department of Research and Development, Norwegian Air Ambulance Foundation, Droebak, Norway; \\ *Corresponding Author: nils.petter.oveland@norskluftambulanse.no \\ ${ }^{2}$ Department of Anesthesiology and Intensive Care, Stavanger University Hospital, Stavanger, Norway \\ ${ }^{3}$ Department of Cardiology, Stavanger University Hospital, Stavanger, Norway \\ ${ }^{4}$ Department of Anesthesiology and Intensive Care, Aarhus University Hospital, Aarhus, Denmark \\ ${ }^{5}$ Faculty of Health Sciences, Institute of Clinical Medicine, Aarhus University, Aarhus, Denmark
}

Received 24 March 2013; revised 25 April 2013; accepted 15 May 2013

Copyright (C) 2013 Nils Petter Oveland et al. This is an open access article distributed under the Creative Commons Attribution License, which permits unrestricted use, distribution, and reproduction in any medium, provided the original work is properly cited.

\begin{abstract}
The clinical assessment of patients with respiratory and circulatory problems can be complex, time consuming and have a high incidence of error. Bedside transthoracic ultrasound (US) is a useful adjunctive test in the evaluation of acutely unstable patients. This case series describes the use of the Focus Assessed Transthoracic Echocardiography (FATE) protocol to diagnose unsuspected pleural collections of fluid and how drainage significantly contributes to the haemodynamic improvement seen in these patients.
\end{abstract}

Keywords: Pleural Effusions; Haemodynamic Instability; Haemothorax; Transthoracic Echocardiography

\section{INTRODUCTION}

The physical examination is the basis for treatmentdecisions of hospitalized critical ill patients often supplemented with measurements of hearth rate, respiratory rate, pulse oxymetry and arterial blood pressure. Depending on the clinical scenario, additional diagnostic tests and procedures such as 12-lead electrocardiogram (ECG), chest X-ray (CXR), arterial blood gas analysis, central venous catheter and pulmonary artery catheter insertions are applied. The resuscitative actions from the treating physicians are commonly intravascular fluid adjustments and/or pharmacological inotropic support [1]. This approach is successful in the majority of cases as many haemodynamic unstable patients benefit from in- creased preload and have some reserve myocardial contractility, which can be improved by inotropic agents. However, this primary survey may lack sufficient diagnostic finesse is subgroups of patients, resulting in misdiagnosis and poorly directed therapy.

Pleural effusions are an under-recorded contributor to haemodynamic instability in intensive care units (ICUs). Experimental physiological models (i.e., using animals) have shown that bilateral pleural effusions produce right ventricular end diastolic collapse and changes in cardiac output that mimic a large pericardial effusion [2]. Furthermore, pleural collections can increase the negative circulatory effect of even small pericardial effusions [3]. Several studies have reported improved haemodynamic parameters following drainage of these pleural effusions with ranging volumes between $500 \mathrm{~mL}$ and $2000 \mathrm{~mL}$ [4-8].

Traditionally anteroposterior CXR has been used to detect pleural fluid collections. This method is timeconsuming and less sensitive when the picture is taken in the supine position [9], whereas ultrasound (US) imaging techniques can reliably detect pleural effusions as small as $100 \mathrm{~mL}$ in the supine position (Figure 1) [10]. In the past two decades there has been an increasing use of point-of-care US (i.e., scanning performed by the treating physicians at bedside). The Focus Assessed Transthoracic Echogardiography (FATE) protocol [10], the Focused Assessment with Sonography for Trauma (FAST) protocol [11], the extended FAST protocol [12], the Undifferentiated Hypotensive Patient (UHP) protocol [13], the Abdominal and Cardiac Evaluation with Sonography in shock (ACES) [14] and the Focused Echocardiographic Evaluation in Resuscitation (FEER) [15] are 
examples of goal-directed US examinations to rapidly diagnose or exclude different causes of haemodynamic instability. Of these, only the FATE and the extended FAST protocols include examinations of the pleural spaces for gross pathology. The FATE protocol consists of three transthoracic cardiac windows and bilateral pleural views (Figure 2). The aim is to assess cardiac function including contractility, chamber size, wall thickness, valve dysfunction and to detect significant pericardial and pleural effusions. Experienced practitioners can perform a full FATE examination in less than 70 seconds with the patient either in supine position or sitting position giving flexibility in different clinical scenarios [10, 16,17].

Pleural effusion may cause severe haemodynamic compromise, but is often missed in the initial evaluation of critically ill patients. Therefore, we here present five patients with respiratory and circulatory problems where rapid insertion of chest tubes and subsequent drainage of pleural collections were associated with a clear improvement in haemodynamic parameters.

\section{CASE SERIES}

\subsection{Case 1}

A 22 months old girl $(11 \mathrm{Kg})$, with no previously diagnosed illness, had the last four weeks experienced episodes of dyspnoea, periods of lethargy and days of vomiting, sweating and cold extremities. En route to the emergency medical service she went into respiratory arrest with possible cardiac arrest inside the car of her parents. Basic life support was initiated and continued by paramedics and an emergency physician under transport to the local hospital's emergency department (i.e., a

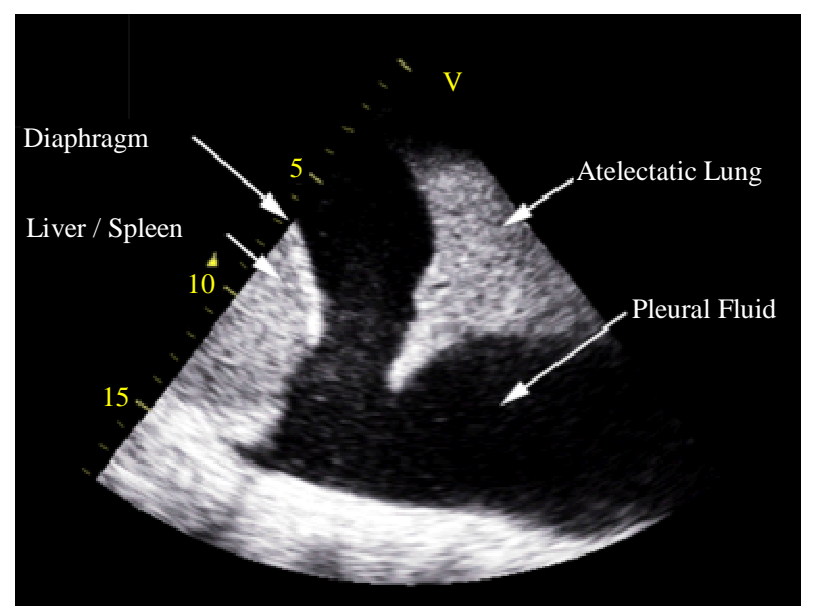

Figure 1. A FATE examination of the right pleural cavity performed with a cardiac probe. The pleural fluid is easy to differentiate from other tissue, as it appears black. Large amounts pleural effusion is often accompanied by atelectasis, nicely illustrated in the figure.
Focus Assessed Transthoracic Echo (FATE)

Scanning through position 1-4 in the most favourable sequence

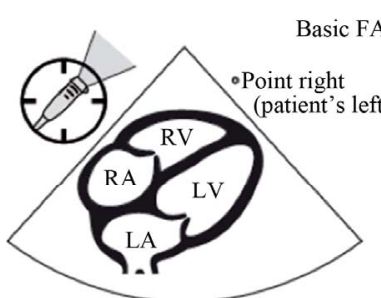

Pos 1: Subcostal 4-chamber

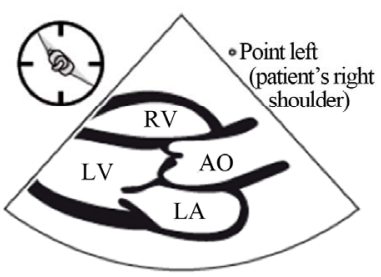

Pos 3: Parasternal long axis

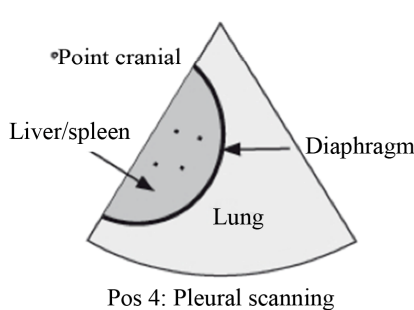

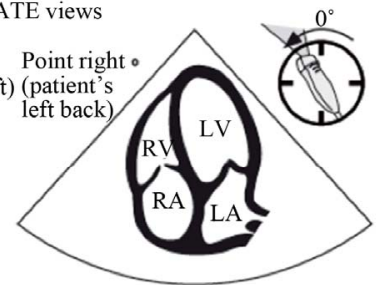

Pos 2: Apical 4-chamber

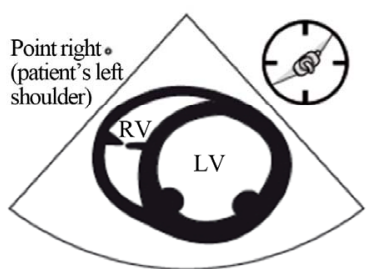

Pos 3: Parasternal LV short axis

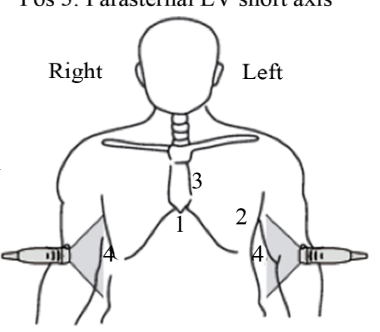

Figure 2. The FATE card (front page) can be requested free of charge at www.fate-protocol.com and are also available as a free application for Android and iPhone.

transport time of three minutes). At arrival, she had return of spontaneous breathing with pronounced stridor, expiratory wheezes, sub-costal retractions and tachypnoea. The skin was pale with capillary refill-time of five seconds, the extremities were cold with weaken distal pulses in the wrists and groins. Initial actions were vascular access in the right external jugular vein, an injecttion of the muscle relaxation agent suxamethonium chloride followed by a rapid sequence endotracheal intubation procedure to secure the airway. At that time the pulse oxymetry was not measurable, the heart rate 130 beats per minute, blood pressure in the right arm 100 over $60 \mathrm{mmHg}$, no palpable pulses in the femoral arteries and the core temperature 36.0 degrees Celsius. One hundred and twenty millilitres (i.e., approximately $10 \mathrm{~mL} / \mathrm{Kg}$ ) of isotonic sodium chloride was subsequently injected based on the clinical suspicion that the girl had severe hypovolemia and sepsis syndrome. The fluid resuscitation precipitated an acute pulmonary oedema worsening the clinical status. The anaesthesiologist on-call performed a FATE examination that revealed a dilated left ventricle with extremely poor contractility, an enlarged right ventricle with pulmonary systolic pressure of 60 $\mathrm{mmHg}$ and bilateral pleural effusions. Chest tubes were inserted and $400 \mathrm{~mL}$ of clear fluid was drained from the 
right pleural cavity and $100 \mathrm{~mL}$ from the left cavity, respectively. After this treatment, the size of right ventricle was reduced and the pulmonary systolic pressure normalized. The more comprehensive transthoracic echocardiography done by the cardiologist showed a dilated left ventricle with an estimated ejection fraction of $15 \%$ $20 \%$, mild mitral regurgitation and a normal aortic arch with no aortic valve pathology. The arterial line in the right femoral artery showed a pressure close to zero, and the final diagnosis of a fused aortic arch (i.e., coarctatio aortae) was confirmed on a computed tomography scan. An air ambulance helicopter brought the child to a tertiary paediatric hospital for corrective surgery the following day.

\subsection{Case 2}

A 48 years old male with New York Heart Association class IV heart failure awaiting cardiac transplant was admitted to the ICU for circulatory and respiratory optimization before surgery. He had a systolic blood pressure of $80 \mathrm{mmHg}$ and oxygen saturation of $88 \%$ on room air. The cardiac index was $1.6 \mathrm{~L} / \mathrm{min} / \mathrm{m}^{2}$ and the mixed venous oxygen saturation $48 \%$. The most recent transthoracic echocardiography four weeks earlier showed a dilatation of the left ventricle with reduced contractility. The FATE examination performed by the ICU physician confirmed earlier findings of a large dilated left ventricle with an ejection fraction of $10 \%$ and no significant pericardial effusion. They did however diagnose a previously unreported right-sided pleural effusion. After insertion of a pleural catheter, $2300 \mathrm{~mL}$ yellow coloured fluid was drained. The cardiac index increased to $1.9 \mathrm{~L} / \mathrm{min} / \mathrm{m}^{2}$, mixed venous oxygen saturation to $65 \%$ and oxygen saturation to $100 \%$. No further therapy was added.

\subsection{Case 3}

A 36 years old male with cardiac failure due to a newly diagnosed dilated cardiomyopathy was referred to a tertiary cardiac centre for further treatment. He was intubated, mechanically ventilated and kept sedated under transport. The inotropic therapy consisted of dopamine $20 \mathrm{mg} / \mathrm{Kg} / \mathrm{min}$, adrenaline $0.03 \mathrm{mg} / \mathrm{kg} / \mathrm{min}$ and noradrenaline $0.08 \mathrm{mg} / \mathrm{kg} / \mathrm{min}$. The transthoracic echocardiography performed by the cardiologist on arrival showed a dilated left ventricle, global hypo-kinesis of the left ventricle with an ejection fraction of only 8\%. During the first 24 hours in the ICU his dopamine was removed and adrenaline and noradrenaline adjusted 1.1 $\mathrm{mg} / \mathrm{kg} / \mathrm{min}$ and $0.23 \mathrm{mg} / \mathrm{kg} / \mathrm{min}$, respectively. The FATE examination the following day showed no change in the left ventricle, but did diagnose an impaired right ventricle function and a moderate right-sided pleural effusion. Percutaneous drainage of $1600 \mathrm{~mL}$ of pleural fluid gave an immediate rise in invasive blood pressure from 83 over $50 \mathrm{mmHg}$ to 97 over $60 \mathrm{mmHg}$ and an improvement in cardiac index from $1.6 \mathrm{~L} / \mathrm{min} / \mathrm{m}^{2}$ to $2.0 \mathrm{~L} / \mathrm{min} / \mathrm{m}^{2}$. The patient was weaned from inotropic support the following week.

\subsection{Case 4}

A 25 years old male that earlier was operated for hollow-chest was anesthetized for removal of the metallic bars in his anterior chest wall. The procedure was technically difficult and after transfer to the post-anaesthesia care unit the patient became unconscious with low heart rate and low blood pressure (i.e., a pulse under 50 and systolic blood pressure of $60 \mathrm{mmHg}$ ). The patient did recover after leg raise (i.e., Trendelenburg position), one mg of intravenous atropine and intravenous fluids. The 12-lead ECG was normal, but the cardiologist on-call still performed a traditional transthoracic echogradiography without including pleural views. Based on the normal findings of the US examination of the heart, the cardiologist together with the surgeon and anaesthesiologist concluded that the event was likely a vagal reaction. Over the following 90 minutes the patient became haemodynamic unstable and was reviewed again by the anaesthesiologist. A CXR showed a possible collection of a fluid in the right pleural cavity, later confirmed by the FATE examination. The cardiac function was normal but a large right-sided haemothorax was diagnosed. The patient returned to operating room for a tube thoracotomy and $1500 \mathrm{~mL}$ blood was evacuated. The drainage combined with the appropriate fluid/blood resuscitation saved the patient from exsanguination, and he was discharged to the ward with no further adverse effects.

\subsection{Case 5}

A 73 years old male with a history of chronic bronchitis was admitted to the thoracic surgery unit for a leftsided post pneumonic empyema. The treatment consisted of antibiotic therapy, chest drainage with a small-sized pigtail catheter and intermittent pleural streptokinase therapy. The amount of bacterial fluid from the catheter was modest, approximating $150 \mathrm{~mL}$ per day for four consecutive days. The control CXRs demonstrated regression but no complete resolution of the empyema. On the fifth day he experienced tachypnea (i.e., 40 breaths per minute), arterial saturation levels of $75 \%$ while receiving supplements of oxygen, hypotension (85 over 60 mmHg), tachycardia (122 heartbeats per minute) with peripheral vasoconstriction. The clinical suspicion was bronchospasm and the patient received inhalations beta-2 agonists and injections of diuretics without obvious effect. The CXR, taken only minutes after his deterioration, revealed left-sided pleural fluid. The patient was then transferred to the operating room where the anaesthetist 
on-call performed a pre-operative FATE examination; the left-sided pleural effusion gave diastolic collapse of the right heart chambers and further compressed the left ventricle. The result was a vast reduction of the hearths contractility. The initial treatment attempt with chest tube failed as it was clotted by coagulated blood. A thoracotomy was planned, but before induction of anaesthesia the patient became more and more bradycardic before going into cardiac arrest with ventricular fibrillation. Cardio-pulmonary resuscitation was commenced, DC electro-shock administered and an acute lateral thoracotomy performed. Approximately $500 \mathrm{~mL}$ of liquid blood and $2500 \mathrm{~mL}$ of clotted blood was removed manually before return of spontaneous circulation. The patient was subsequently transferred to the ICU with an adrenaline infusion of $0.06 \mathrm{mg} / \mathrm{kg} / \mathrm{min}$. Unfortunately, the patient did not make full neurological recovery at discharge.

\section{DISCUSSION}

These five hemodynamic unstable patients all showed substantial clinical improvement after the detection and treatment of their pleural fluid collections. The cases can be divided into two groups; those patients with advanced cardiovascular pathology with secondary pleural effusions and those patients with a primary thoracic condition with pleural fluid/blood causing their instability.

In case scenario one to three, the drainage of pleural fluid stabilised the patients sufficiently to enable subsequent definitive management. In case number one; the child was initially treated for suspected septic shock with an intravenous bolus of isotonic fluid that led to fulminant pulmonary oedema. The diagnosis based on the clinical examination was wrong. In fact, the aortic arch was blocked leading to left ventricular heart failure, bilateral pleural effusions, respiratory distress and eventual respiratory and circulatory collapse. Early detection and drainage of her pleural effusions provided initial stabilisation prior to definitive surgical management. The patient in case number two suffered from chronic cardiac failure complicated by pleural effusion. His clinical deterioration had been gradual and the treating physicians became fixated on his cardiac performance. At the same time they overlooked the pleural fluid that was exacerbating his clinical state. More timely identification and drainage of the pleural cavity may have stopped his deterioration and prevented the admission to the ICU. In case number three; the patient also improved dramatically following the percutaneous drainage procedure. If a FATE scan had been performed prior to the transfer between hospitals, insertion of chest tubes may have optimized the patient's clinical status before transport. These clinical scenarios are complex and show diversity in the causes of circulatory instability. The stabilizing effect of pleural drainage is eminent, but the diagnosis of the effu- sions and thus the definitive treatment was unnecessarily delayed in the three case reports.

In the last two patients (i.e., case number four and five), a combination of tunnel vision by the treating physicians and the time delay in obtaining CXRs prevented a prompt and accurate diagnosis and management of large haemorrhages in the pleural cavity. In case number four: the patient's clinical deterioration was due to a concealed bleeding into the chest caused by removal of metallic bars after a hollow-chest operation. The first postoperative transthoracic echocardiography was done to exclude cardiac injury and tamponade, but provided false reassurance that there was nothing wrong with the patient. This emphasizes the hazard of having a "single organ" approach in critical care. Taking this into account, a FATE examination at the time of the patient's initial instability would most likely have detected the haemothorax and expedited tube thoracostomy. In case number five; futile beta- 2 agonist inhalations and diuretic therapy failed and the patient condition rapidly deteriorated. To obtain the CXR that showed a massive pleural bleeding, valuable time went before any measures were taken. A point-of-care US examination of the heart and pleural would have ensured earlier diagnosis and definitive treatment.

\section{CONCLUSION}

Regardless of the cause of respiratory and circulatory collapse, a prompt and correct diagnosis of the patient's condition is paramount. Despite being less sensitive than thoracic US, CXRs are still the most common adjunct in the initial evaluation of critical ill patients. To reduce the risk of error, point-of-care US assessments of the pleural cavity should be incorporated into the primary survey of respiratory and circulatory unstable patients. The FATE protocol is a comprehensive, rapid and non-invasive examination aimed to find potential causes of haemodynamic instability in critically ill patients. FATE also includes US imaging of the pleural cavity to avoid that life-threatening pleural collections are missed. The protocol is easy to learn and sonographers can download all the essential elements of the "FATE Card" as a free application for Android and iPhone illustrated in Figure 2.

\section{ACKNOWLEDGEMENTS}

The authors acknowledge the five patients and their next of kin for allowing us to tell their stories and The Norwegian Air Ambulance Foundation for financing the publication fee.

\section{REFERENCES}

[1] Oldner, A., Rossi, P., Karason, S. and Aneman, A. (2003) A practice survey on vasopressor and inotropic drug 
therapy in Scandinavian intensive care units. Acta Anaesthesiologica Scandinavica, 47, 693-701. doi:10.1034/j.1399-6576.2003.00129.x

[2] Wemmelund, K., Lie, R.H., Juhl-Olsen, P., Frederiksen, C.A., Hermansen, J.F. and Sloth, E. (2012) Pleural effusion decreases left ventricular pre-load and causes haemodynamic compromise: An experimental porcine study. Acta Anaesthesiologica Scandinavica, 56, 833-839. doi:10.1111/j.1399-6576.2012.02678.x

[3] Traylor, J.J., Chan, K., Wong, I., Roxas, J.N. and Chandraratna, P.A. (2002) Large pleural effusions producing signs of cardiac tamponade resolved by thoracentesis. The American Journal of Cardiology, 89, 106-108. doi:10.1016/S0002-9149(01)02180-4

[4] Alam, H.B., Levitt, A., Molyneaux, R., Davidson, P. and Sample, G.A. (1999) Can pleural effusions cause cardiac tamponade? Chest, 116, 1820-1822. doi:10.1378/chest.116.6.1820

[5] Kaplan, L.M., Epstein, S.K., Schwartz, S.L., Cao, Q.L. and Pandian, N.G. (1995) Clinical, echocardiographic, and hemodynamic evidence of cardiac tamponade caused by large pleural effusions. American Journal of Respiratory and Critical Care Medicine, 151, 904-908. doi:10.1164/ajrccm/151.3_Pt_1.904

[6] Kisanuki, A., Shono, H., Kiyonaga, K., Kawataki, M., Otsuji, Y., Minagoe, S., Nakao, S., Nomoto, K. and Tanaka, H. (1991) Two-dimensional echocardiographic demonstration of left ventricular diastolic collapse due to compression by pleural effusion. American Heart Journal, 122, 1173-1175. doi:10.1016/0002-8703(91)90494-3

[7] Kopterides, P., Lignos, M., Papanikolaou, S., Papadomichelakis, E., Mentzelopoulos, S., Armaganidis, A. and Panou, F. (2006) Pleural effusion causing cardiac tamponade: report of two cases and review of the literature. Heart \& Lung: The Journal of Critical Care, 35, 66-67. doi:10.1016/j.hrtlng.2005.07.001

[8] Negus, R.A., Chachkes, J.S. and Wrenn, K. (1990) Tension hydrothorax and shock in a patient with a malignant pleural effusion. The American Journal of Emergency Medicine, 8, 205-207. doi:10.1016/0735-6757(90)90323-R

[9] Woodring, J.H. (1984) Recognition of pleural effusion on supine radiographs: How much fluid is required? AJR American Journal of Roentgenology, 142, 59-64.

[10] Jensen, M.B., Sloth, E., Larsen, K.M. and Schmidt, M.B.
(2004) Transthoracic echocardiography for cardiopulmonary monitoring in intensive care. European Journal of Anaesthesiology, 21, 700-707. doi:10.1017/S0265021504009068

[11] Scalea, T.M., Rodriguez, A., Chiu, W.C., Brenneman, F.D., Fallon Jr., W.F., Kato, K., McKenney, M.G., Nerlich, M.L., Ochsner, M.G. and Yoshii, H. (1999) Focused assessment with sonography for trauma (FAST): Results from an international consensus conference. The Journal of Trauma, 46, 466-472. doi:10.1097/00005373-199903000-00022

[12] Kirkpatrick, A.W., Sirois, M., Laupland, K.B., Liu, D., Rowan, K., Ball, C.G., Hameed, S.M., Brown, R., Simons, R., Dulchavsky, S.A., et al. (2004) Hand-held thoracic sonography for detecting post-traumatic pneumothoraces: The extended focused assessment with sonography for trauma (EFAST). The Journal of Trauma, 57, 288-295. doi:10.1097/01.TA.0000133565.88871.E4

[13] Rose, J.S., Bair, A.E., Mandavia, D. and Kinser, D.J. (2001) The UHP ultrasound protocol: A novel ultrasound approach to the empiric evaluation of the undifferentiated hypotensive patient. The American Journal of Emergency Medicine, 19, 299-302. doi:10.1053/ajem.2001.24481

[14] Atkinson, P.R., McAuley, D.J., Kendall, R.J., Abeyakoon, O., Reid, C.G., Connolly, J. and Lewis, D. (2009) Abdominal and cardiac evaluation with sonography in shock (ACES): An approach by emergency physicians for the use of ultrasound in patients with undifferentiated hypotension. Emergency Medicine Journal, 26, 87-91. doi:10.1136/emj.2007.056242

[15] Breitkreutz, R., Walcher, F. and Seeger, F.H. (2007) Focused echocardiographic evaluation in resuscitation management: Concept of an advanced life support-conformed algorithm. Critical Care Medicine, 35, S150-S161. doi:10.1097/01.CCM.0000260626.23848.FC

[16] Frederiksen, C.A., Juhl-Olsen, P., Larsen, U.T., Nielsen, D.G., Eika, B. and Sloth, E. (2010) New pocket echocardiography device is interchangeable with high-end portable system when performed by experienced examiners. Acta Anaesthesiologica Scandinavica, 54, 1217-1223. doi:10.1111/j.1399-6576.2010.02320.x

[17] Frederiksen, C.A., Knudsen, L., Juhl-Olsen, P. and Sloth, E. (2011) Focus-assessed transthoracic echocardiography in the sitting position: Two life-saving cases. Acta Anaesthesiologica Scandinavica, 55, 126-129. doi:10.1111/j.1399-6576.2010.02330.x 